\title{
Volumen mediante percepción clínica y bioimpedancia: el hombre o la máquina
}

\author{
Nemesio M. Martínez Martínez, Lourdes de la Vara Iniesta, Gonzalo Martínez Fernández, Cristina Pérez \\ Jiménez, Encarnación Bellón Pérez, Juan Pérez Martínez
}

\section{S. Nefrología. Complejo Hospitalario Universitario. Albacete}

\section{Introducción:}

Diversos estudios confirman que una sobrehidratación continua está asociada con mayor morbimortalidad. Todo ello indica que es conveniente utilizar en nuestra rutina herramientas eficaces, sencillas y precisas para la determinación del estado de hidratación de nuestros pacientes. Los profesionales sanitarios somos capaces de estimarlo mediante nuestra percepción clínica, pero, además, gracias a otras herramientas como la bioimpedancia, es posible analizar la composición corporal y el estado de hidratación de una manera más objetiva.

\section{Objetivo:}

Determinar si existen diferencias entre el estado de hidratación estimado mediante bioimpedancia, frente a la percepción clínica de los profesionales sanitarios de nuestra Unidad de Diálisis Peritoneal.

\section{Material y método:}

Estudio descriptivo observacional realizado en nuestra Unidad de Diálisis Peritoneal, incluyendo 37 pacientes durante un periodo de 4 meses. Se ha comparado la estimación de volumen de sobrehidratación estimado mediante bioimpedancia y, de manera ciega, mediante la percepción clínica de los distintos profesionales de nuestra unidad (4 enfermeros, 1 médico adjunto, 1 médico residente). Se consideró como criterio de exclusión aquellos pacientes porta- dores de elementos metálicos no extraíbles y negativa a participar en el estudio. Los datos se analizaron con el paquete estadístico SPSS $®$ V.15.0. Los datos se presentan como proporciones, media \pm desviación estándar. Las comparaciones entre estimadores centrales se realizaron mediante el test de Student. La fiabilidad de las medidas se evaluó mediante el gráfico de Bland-Altman, realizado con el software MedCalc $® v \cdot 12.5 .0$. Resultados y discusión: Se analizaron 111 determinaciones. La media de sobrehidratación estimada por bioimpedancia fue de $2,77 \mathrm{~L}(+/-$ $2,05 \mathrm{~L})$, un $1,0054 \%(+/-0,8676 \%)$ respecto al Vurea. La media de sobrehidratación estimada por los profesionales sanitarios fue de $2,04 \mathrm{~L}(+/-1,67 \mathrm{~L})$, un $0,76 \%(+/-0,71 \%)$ respecto al volumen de distribución $(\mathrm{V})$. La correlación entre estos dos métodos es significativa $(p=0,035)$. Nos desviamos frente a la bioimpedancia de media $-0,24 \%(+/-0,11 \%)$, por lo que tendemos a infraestimar el estado de hidratación de nuestros pacientes.

\section{Conclusiones:}

Encontramos una alta correlación entre el estado de hidratación estimado por la percepción clínica de los profesionales sanitarios y la bioimpedancia en pacientes en diálisis peritoneal y, aunque la bioimpedancia puede ayudar al clínico a identificar pequeñas modificaciones en la volemia de nuestros pacientes de una manera más objetiva, no sustituye la percepción clínica del profesional sanitario. 


\section{Referencias Bibliográficas}

1. Arias M. La bioimpedancia como valoración del peso seco y del estado de hidratación. Dial Trasp. 2010; 31(4): 137-139.

2. Caravaca F, Martínez del Viejo. Estimación del estado de hidratación mediante bioimpedancia es- pectroscópica multifrecuencia en la enfermedad renal avanzada. Nefrología 2011; 31 (5): 537-44.

3. Juan García I. Integración de la bioimpedancia multifrecuencia, parámetros ecocardiográficos y bioquímicos en la valoración y manejo del estado de hidratación de pacientes en diálisis. Tesis doctoral (2012). 